\title{
Triethylborane-induced free radical reactions with benzylidene Meldrum's acids. Simple and efficient synthesis of benzyl Meldrum's acids
}

\author{
Shijay Gao, Tingkai Tzeng, Ying-Tsang Lan, Ju-Tsung Liu, and Ching-Fa Yao* \\ Department of Chemistry, National Taiwan Normal University 88, Sec. 4, Tingchow Road, Taipei, \\ Taiwan 116 ROC \\ E-mail: cheyaocf@ntnu.edu.tw
}

\begin{abstract}
We have developed a simple aerobic synthesis of benzyl Meldrum's acids from benzylidene Meldrum's acids with $\mathrm{Et}_{3} \mathrm{~B}$ or $\mathrm{RI} / \mathrm{Et}_{3} \mathrm{~B}$. The reaction system has also been applied successfully to the synthesis of benzyl Meldrum's acids in a one-pot procedure.
\end{abstract}

Keywords: Triethylborane, free radical, Meldrum's acid, benzyl Meldrum's acid, benzylidene Meldrum's acid

\section{Introduction}

The conjugate addition reaction of organometallic reagents to $\alpha, \beta$-unsaturated systems is one of the most widely used synthetic methods for carbon-carbon bond formation. ${ }^{1}$ Recently, reactions of $\alpha, \beta$ unsaturated systems with various kinds of organometallic reagents derived from lithium, ${ }^{2}$ magnesium, ${ }^{3}$ zinc, ${ }^{4}$ aluminum, ${ }^{5}$ copper, ${ }^{6}$ and boronate ${ }^{7}$ have been reported. Similarly, a catalytic asymmetric conjugate addition promoted by chiral metal complexes also has been shown to be an efficient method for enantioselective carbon-carbon bond formation. ${ }^{6}$ In the majority of these reactions, the carbon nucleophile is an ionic species and most often is an organocopper reagent. Despite extensive literature on the ionic reaction, comparatively little research has focused on the free radical variation. It is only quite recently that the conjugate addition of free radicals to $\alpha, \beta$ unsaturated systems has been successfully studied by using tin hydride as the radical initiator. ${ }^{8}$ Carbon-carbon bond formation via a free radical-mediated reaction has led to a variety of useful applications in organic synthesis. ${ }^{9}$ In view of the excellent characteristics of triethylborane as a free radical initiator in aqueous solution and under aerobic conditions, we were prompted to examine the feasibility of $\mathrm{Et}_{3} \mathrm{~B}$-mediated free radical functionalized reactions of benzylidene Meldrum's acids prepared by Knoevenagel condensation of Meldrum's acid (2,2-dimethyl-1,3-dioxane-4,6-dione) and aldehydes under economical and eco-friendly conditions. Recently, Meldrum's acid derivatives have gained considerable attention not only from the novel chemistry they exhibit but also from the varied transformations they undergo. ${ }^{10}$ Meldrum's acid derivatives are key synthetic building 
blocks, with 5 -alkylated, ${ }^{11}$ 5-acyl, ${ }^{12}$ 5-benzylidene, ${ }^{13}$ and 5 -benzyl derivatives ${ }^{14}$ capable of subsequently useful transformations. In this paper, we wish to combine the feasibility of triethylborane with the synthetic utility of Meldrum's acid derivatives to develop a simple and effective procedure for the synthesis of various benzyl Meldrum's acid.

\section{Results and Discussion}

Based on our previous studies, ${ }^{15}$ the results of benzyl Meldrum's acid derivatives obtained by reacting benzylidene Meldrum's acid with $\mathrm{Et}_{3} \mathrm{~B}$ or $\mathrm{RI} / \mathrm{Et}_{3} \mathrm{~B}$ are shown in Scheme 1.

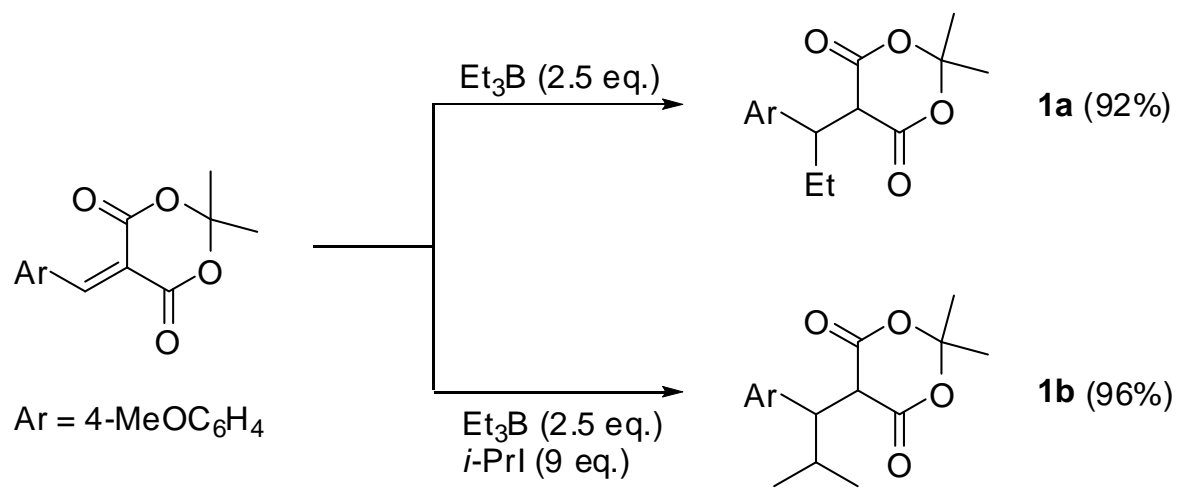

Scheme 1. Reaction of benzylidene Meldrum's acid with $\mathrm{Et}_{3} \mathrm{~B}$ and RI.

It is noteworthy to observe that corresponding benzyl Meldrum's acids 1b were obtained in excellent yield. Our previous studies found that the treatment of dimethyl benzylidenemalonate with $\mathrm{RI} / \mathrm{Et}_{3} \mathrm{~B}$ not only affords the $\beta$-monoalkylated but also the $\alpha, \beta$-dialkylated product at the same time. ${ }^{15 \mathrm{e}}$ Although the quantity of $\alpha, \beta$-dialkylated product could been limited by using triethylaluminum, the air-sensitivity of this reagent makes it very difficult to handle and leads to a tedious to workup. The different results are explicable from the different coordination abilities of triethylborane and triethylaluminum. The size of the aluminum atom facilitates a tighter coordination to the oxygen of the dimethyl benzylidenemalonate than is the case for boron so that the substrates could be activated more efficiently and could be attacked by the nucleophilic alkyl radical easily. Comparatively, in the reaction of benzylidene Meldrum's acid 1 with $\mathrm{RI} / \mathrm{Et}_{3} \mathrm{~B}$ no $\alpha, \beta-$ dialkylated product was observed. We proposed that the steric hindrance maybe plays an important role to restrict the formation of $\alpha, \beta$-dialkylated products. As shown in Table 1, the free radical mediated-conjugate addition could be carried out with various benzylidene Meldrum's acids in 40$96 \%$ yields by using $\mathrm{Et}_{3} \mathrm{~B}$ and various alkyl iodides. Both aromatic and aliphatic benzylidene Meldrum's acids reacted with various alkyl radicals, induced by triethylborane, and afford the corresponding benzyl Meldrum's acids in good to excellent yields. The yields of benzyl Meldrum's acid carrying electron-withdrawing groups were lower than those with electron-donating groups and this is ascribed to a poorer solubility under the reaction conditions. 


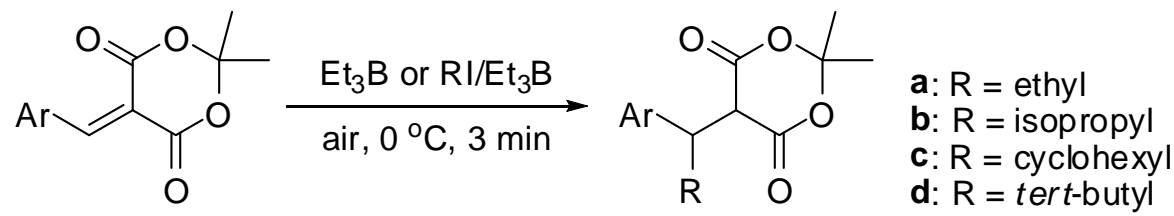

Table 1. The synthesis of benzyl Meldrum's acids from benzylidene Meldrum's acid and $\mathrm{Et}_{3} \mathrm{~B}$ or $\mathrm{RI} / \mathrm{Et}_{3} \mathrm{~B}^{\mathrm{a}}$

\begin{tabular}{|c|c|c|c|c|c|}
\hline Entry & $\mathrm{Ar}$ & RI (eq.) & $\begin{array}{l}\mathrm{Et}_{3} \mathrm{~B} \\
\text { (eq.) }\end{array}$ & $\begin{array}{c}\text { Product } \\
\text { code }\end{array}$ & Yield $(\%)^{b}$ \\
\hline 1 & & - & 2.5 & $1 a$ & 92 \\
\hline 2 & & $\mathrm{R}=i-\operatorname{Pr}(9)$ & 2.5 & $1 b$ & 96 \\
\hline 3 & & $\mathrm{R}=c-\mathrm{C}_{6} \mathrm{H}_{11}(9)$ & 2.5 & 1c & 86 \\
\hline 4 & & $\mathrm{R}=t-\mathrm{Bu}(6)$ & 2.5 & 1d & 96 \\
\hline 5 & & - & 2.5 & $2 a$ & 80 \\
\hline 6 & & $\mathrm{R}=i-\operatorname{Pr}(9)$ & 2.5 & $2 \mathbf{b}$ & 80 \\
\hline 7 & & $\mathrm{R}=c-\mathrm{C}_{6} \mathrm{H}_{11}(9)$ & 2.5 & $2 c$ & 95 \\
\hline 8 & & $\mathrm{R}=t-\mathrm{Bu}(6)$ & 2.5 & 2d & 87 \\
\hline 9 & & - & 2.5 & 3a & 89 \\
\hline 10 & & $\mathrm{R}=i-\operatorname{Pr}(9)$ & 2.5 & $3 \mathbf{b}$ & 84 \\
\hline 11 & & $\mathrm{R}=c-\mathrm{C}_{6} \mathrm{H}_{11}(9)$ & 2.5 & $3 c$ & 90 \\
\hline 12 & & $\mathrm{R}=t-\mathrm{Bu}(6)$ & 2.5 & 3d & 95 \\
\hline 13 & & - & 2.5 & $4 a$ & 56 \\
\hline 14 & & $\mathrm{R}=i-\operatorname{Pr}(9)$ & 2.5 & $4 b$ & 40 \\
\hline 15 & & $\mathrm{R}=c-\mathrm{C}_{6} \mathrm{H}_{11}(9)$ & 2.5 & $4 c$ & 40 \\
\hline 16 & & $\mathrm{R}=t-\mathrm{Bu}(6)$ & 2.5 & 4d & 62 \\
\hline 17 & & - & 2.5 & $5 a$ & 77 \\
\hline 18 & & $\mathrm{R}=i-\operatorname{Pr}(9)$ & 2.5 & $5 b$ & 72 \\
\hline 19 & & $\mathrm{R}=c-\mathrm{C}_{6} \mathrm{H}_{11}(9)$ & 2.5 & $5 c$ & 87 \\
\hline 20 & & $\mathrm{R}=t-\mathrm{Bu}(6)$ & 2.5 & $5 d$ & 91 \\
\hline 21 & & - & 2.5 & $6 a$ & 84 \\
\hline 22 & & $\mathrm{R}=i-\operatorname{Pr}(9)$ & 2.5 & $6 b$ & $80^{\mathrm{c}}$ \\
\hline 23 & & $\mathrm{R}=c-\mathrm{C}_{6} \mathrm{H}_{11}(9)$ & 2.5 & $6 c$ & 92 \\
\hline 24 & & $\mathrm{R}=\mathrm{t}-\mathrm{Bu}(6)$ & 2.5 & 6d & 70 \\
\hline
\end{tabular}

${ }^{a}$ The reaction was performed using $1 \mathrm{mmol}$ of benzylidene Meldrum's acid and $2.5 \mathrm{mmol}$ of $\mathrm{Et}_{3} \mathrm{~B}$ or $2.5 \mathrm{mmol} \mathrm{Et}_{3} \mathrm{~B}$ with $\mathrm{RI}$ (selected equiv) in $10 \mathrm{~mL} \mathrm{THF}$ at $0{ }^{\circ} \mathrm{C}$.

${ }^{\mathrm{b}}$ Isolated yield.

${ }^{\mathrm{C}} \mathbf{6 a}$ was also present in $5 \%$ yield. 
The same reaction system has been extended efficiently to iodo-adamantane with triethylborane under similar conditions. Thus, the reaction of benzylidene Meldrum's acid 1 with the 1-adamantyl radical afforded the corresponding derivative 7 in 50\% isolated yield (Scheme 2). The low yield of 7 could be due to steric hindrance of 1-adamantyl radical.

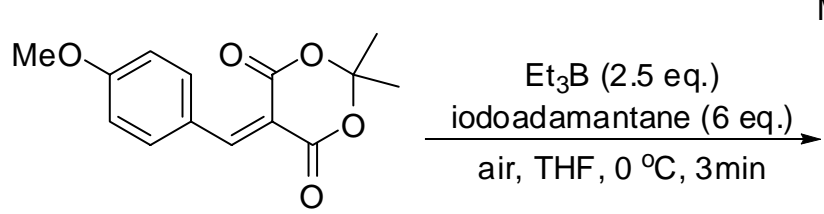

1

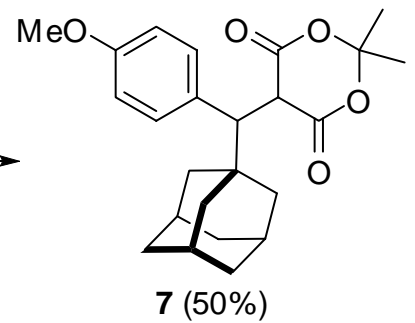

Scheme 2. Reaction of benzylidene Meldrum's acid with the 1-adamantyl radical.

On the basis of literature results and our own studies, ${ }^{15 \mathrm{~g}}$ the reaction system could be successfully applied to the one-pot synthesis of various benzyl Meldrum's acids from $\mathrm{RI} / \mathrm{Et}_{3} \mathrm{~B}$ and benzylidene derivatives prepared in situ from Meldrum's acids and aldehydes. The yields from the one-pot syntheses are shown in Scheme 3.

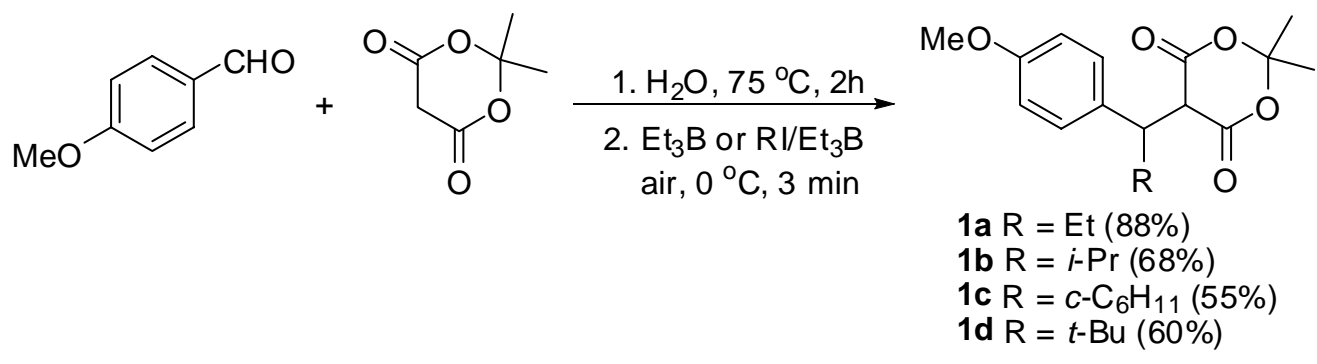

Scheme 3. One-pot synthesis of various benzyl Meldrum's acids.

On the basis of our own studies on the triethylborane-induced free radical chemistry, the results show that oxygen is not only known to be a free radical scavenger but also to be a free radical initiator. ${ }^{15}$ A mechanism for the above reactions is proposed in Scheme 4. 


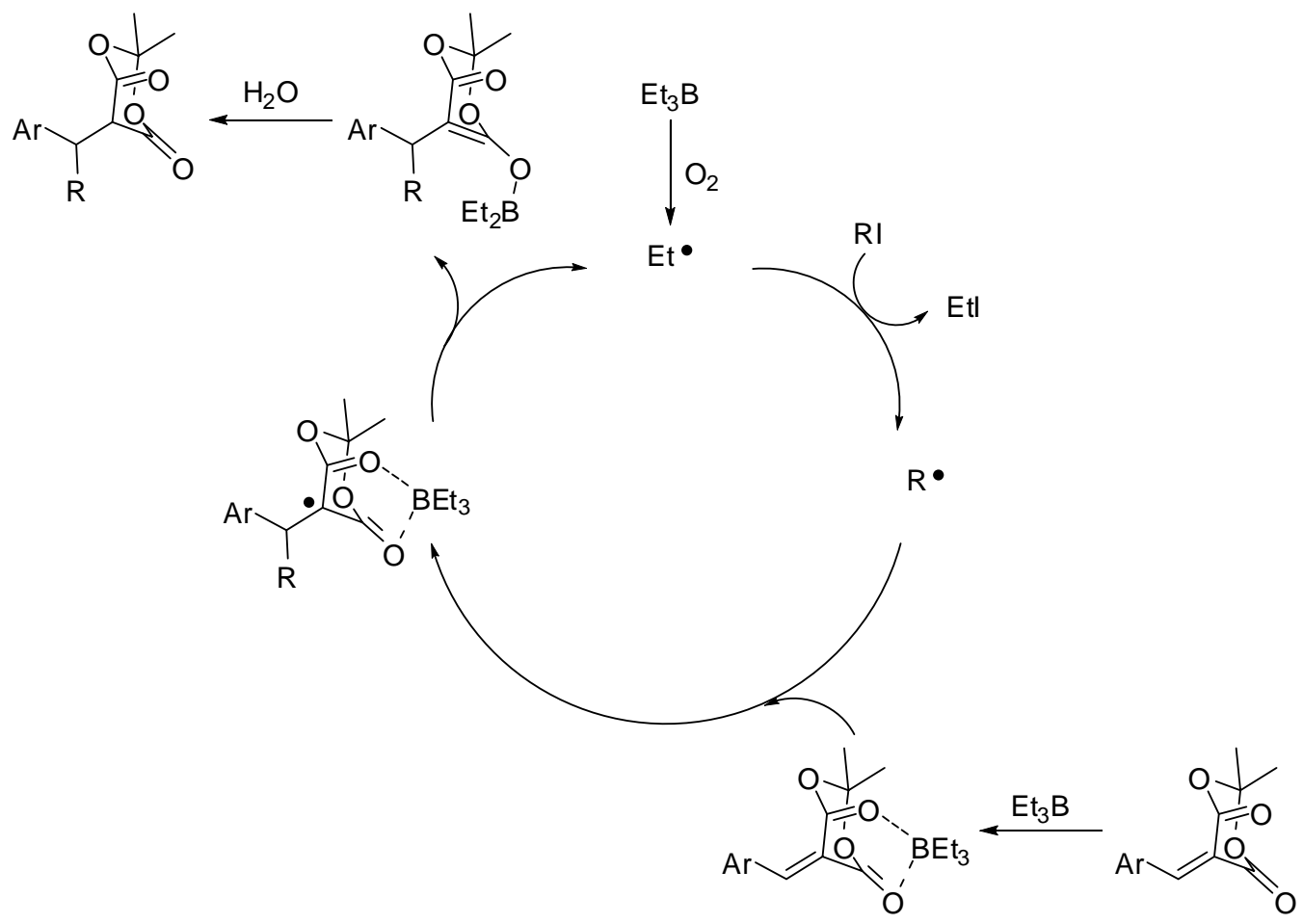

Scheme 4. Proposed mechanism for the synthesis of benzyl Meldrum's acid.

The synthetic utility of the benzyl Meldrum's acid preparations has been extended as shown in Scheme 5 through synthesis of the corresponding 1-indanone derivatives. Initially, the treatment of benzylidene Meldrum's acid with $\mathrm{Et}_{3} \mathrm{~B}$ or $\mathrm{i}-\mathrm{PrI} / \mathrm{Et}_{3} \mathrm{~B}$ at $0{ }^{\circ} \mathrm{C}$ for $3 \mathrm{~min}$ afforded benzyl Meldrum's acids $\mathbf{8 a}$ and $\mathbf{8 b}$ in $92 \%$ and $79 \%$ yields, respectively. These were converted into the corresponding 1 -indanones $9 \mathbf{a}$ and $\mathbf{9 b}$ by $\mathrm{Sc}(\mathrm{OTf})_{3}$ catalysis under reflux in $60 \%$ and $63 \%$ yields, respectively. ${ }^{14}$

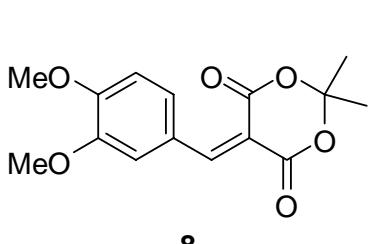

8

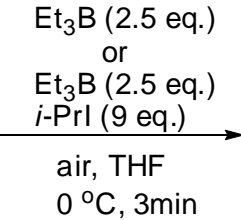

$0{ }^{\circ} \mathrm{C}, 3 \mathrm{~min}$

9a $R=E t(60 \%)$

9b $\mathrm{R}=i-\operatorname{Pr}(63 \%)$

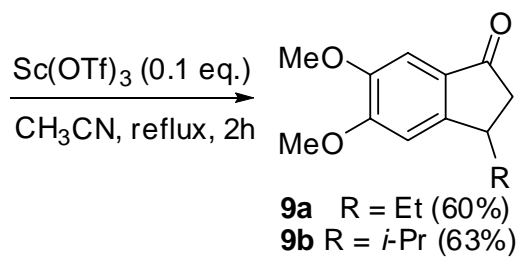

Scheme 5. The synthesis of 1-indanones from benzylidene Meldrum's acids.

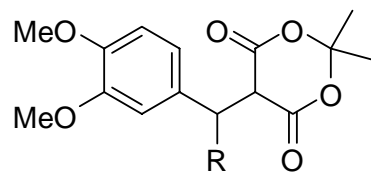

$8 \mathbf{a} \mathrm{R}=\mathrm{Et}(92 \%)$

8b $\mathrm{R}=i-\operatorname{Pr}(79 \%)$ 


\section{Conclusions}

In comparison with our previous $\mathrm{study}^{15 \mathrm{e}}$, we have now found that the formation of the $\alpha, \beta$ dialkylated products cannot only be avoided by using a strong coordinative reagent such as triethylaluminum but also by using sterically hindered benzylidene Meldrum's acids. Herein, we have developed a simple procedure for the synthesis of benzyl Meldrum's acids, which are key building blocks for synthesis. The reaction system was successfully applied to a variety of benzylidene Meldrum's acids as well as alkyl radicals generated by triethylborane. Various benzyl Meldrum's acids have been prepared in a one-pot procedure.

\section{Experimental Section}

General. All reagents and chemicals were purchased from Sigma-Aldrich Chemical Company, Acros organics, Alfa Aesar or Merck and were used as received. Analytical thin layer chromatography was performed with Merck silica gel $60 \mathrm{~F}$ glass plates and flash chromatography by the use of Merck silica gel 60 (230-400 mesh). Melting points were determined on a microscope hot-stage apparatus and are uncorrected. ${ }^{1} \mathrm{H}-\mathrm{NMR}$ and ${ }^{13} \mathrm{C}-\mathrm{NMR}$ spectra were recorded at 400 and $100 \mathrm{MHz}$, respectively, on a Bruker Avance $400 \mathrm{FT}-\mathrm{NMR}$ instrument. Chloroform- $d$ was used as the solvent and TMS $(\delta=0.00)$ as an internal standard. Chemical shift values are reported in ppm relative to TMS. Multiplicities are recorded as s (singlet), $d$ (doublet), t (triplet), q (quartet), dd (doublet of doublet), br (broadened), m (multiplet). Coupling constants $(J)$ are in Hz. MS and HRMS were measured on JEOL JMS-D300 and JEOL JMS-HX110 spectrometers, respectively.

\section{Typical experimental procedures for the synthesis of benzyl Meldrum's acids from benzylidene Meldrum's acids (Table 1)}

In a pyrex test tube with a magnetic stirrer were placed benzylidene Meldrum's acid ( $1 \mathrm{mmol})$ alone or with alkyl iodide (selected equiv) in THF $(10 \mathrm{~mL})$ at $0{ }^{\circ} \mathrm{C}$. Triethylborane $(2.5 \mathrm{mmol})$ was added dropwise into the mixture solution, into which was bubbled air from air pump at $0{ }^{\circ} \mathrm{C}$. After $3 \mathrm{~min}$ the reaction mixture was poured into ice cold water and then extracted with $\mathrm{CH}_{2} \mathrm{Cl}_{2}(3 \times 25 \mathrm{~mL})$. The combined $\mathrm{CH}_{2} \mathrm{Cl}_{2}$ layers were washed with brine, dried over anhydrous $\mathrm{MgSO}_{4}$, and the solvents removed. The crude product was purified by flash column chromatography to obtain benzyl Meldrum's acids $\mathbf{1}$.

\section{Typical experimental procedures for the one-pot synthesis of benzyl Meldrum's acids (Scheme}

3)

In a pyrex test tube with a magnetic stirrer were placed aldehyde $(1.1 \mathrm{mmol})$ and Meldrum's acid (1 $\mathrm{mmol})$ in water $(3 \mathrm{~mL})$ and the whole heated to $75^{\circ} \mathrm{C}$ for $2 \mathrm{~h}$. After cooling to room temperature, THF (10 mL) alone or containing alkyl iodide (selected equiv) was added to the aqueous solution. Triethylborane $(2.5 \mathrm{mmol})$ was added dropwise into the THF-water biphasic solution, into which was bubbled air from air pump at $0{ }^{\circ} \mathrm{C}$. After 3 min the reaction mixture was poured into ice cold 
water and then extracted with $\mathrm{CH}_{2} \mathrm{Cl}_{2}(3 \times 25 \mathrm{~mL})$. The combined $\mathrm{CH}_{2} \mathrm{Cl}_{2}$ layers were washed with brine, dried over anhydrous $\mathrm{MgSO}_{4}$, and the solvents removed. The crude product was purified by flash column chromatography to obtain benzyl Meldrum's acids $\mathbf{1}$.

5-[1-(4-Methoxyphenyl)-propyl]-2,2-dimethyl-1,3-dioxane-4,6-dione 1a. (Table 1, entry 1). Yield 92\% (268 mg); Colorless oil; IR (KBr, neat) $v_{\max }$ 2966, 2937, 1733, 1716, 1612, 1512, 1465, 1395, 1380, 1249, 1177, 1034, 1004, 831, 547; ${ }^{1} \mathrm{H}$ NMR (400 MHz, $\left.\mathrm{CDCl}_{3}\right) \delta$ 7.27-7.19 $(\mathrm{m}, 2 \mathrm{H}), 6.85-6.81(\mathrm{~m}, 2 \mathrm{H}), 3.77(\mathrm{~s}, 3 \mathrm{H}), 3.68(\mathrm{~d}, J=3.2 \mathrm{~Hz}, 1 \mathrm{H}), 3.63-3.58(\mathrm{~m}, 1 \mathrm{H}), 2.31-2.20$ $(\mathrm{m}, 1 \mathrm{H}), 2.12-2.03(\mathrm{~m}, 1 \mathrm{H}), 1.63(\mathrm{~s}, 3 \mathrm{H}), 1.17(\mathrm{~s}, 3 \mathrm{H}), 0.93(\mathrm{t}, J=7.32 \mathrm{~Hz}, .3 \mathrm{H}) ;{ }^{13} \mathrm{C}$ NMR $(100$ $\left.\mathrm{MHz}_{,} \mathrm{CDCl}_{3}\right) \delta 166.27,165.02,159.19,131.60,130.27,114.19,105.60,55.40,51.30,47.65$, 28.63, 28.29, 26.15, 12.78; MS m/z (relative intensity) $292\left(\mathrm{M}^{+}, 4\right), 206$ (76), $190\left[\left({\mathrm{M}-\mathrm{CO}_{2}-}^{-}\right.\right.$ $\left.\left.\mathrm{C}_{3} \mathrm{H}_{6} \mathrm{O}\right)^{+}, 1\right], 188$ (81), 161 (25), 149 (47), 133 (46), 121 (100); HRMS (EI) m/z calcd for $\mathrm{C}_{16} \mathrm{H}_{20} \mathrm{O}_{5}\left(\mathrm{M}^{+}\right)$292.1311, found 292.1306.

5-[1-(4-Methoxyphenyl)-2-methylpropyl]-2,2-dimethyl-1,3-dioxane-4,6-dione 1b. (Table 1, entry 2). Yield 96\% (293 mg); White solid, m.p. 89-90 ${ }^{\circ} \mathrm{C}$; IR (KBr, neat) $v_{\max } 2970,2839$, 1770, 1735, 1608, 1513, 1471, 1454, 1395, 1386, 1298, 1244, 1202, 1177, 1045, 1028, 1003, 828, 752, 541; ${ }^{1} \mathrm{H}$ NMR (400 MHz, $\left.\mathrm{CDCl}_{3}\right) \delta 7.20-7.16(\mathrm{~m}, 2 \mathrm{H}), 6.84-6.80(\mathrm{~m}, 2 \mathrm{H}), 3.87(\mathrm{~d}, J=$ $3.2 \mathrm{~Hz}, 1 \mathrm{H}), 3.77(\mathrm{~s}, 3 \mathrm{H}), 3.24(\mathrm{dd}, J=11.3,3.1 \mathrm{~Hz}, 1 \mathrm{H}), 2.79-2.67(\mathrm{~m}, 1 \mathrm{H}), 1.61(\mathrm{~s}, 3 \mathrm{H}), 1.19$ $(\mathrm{d}, J=6.5 \mathrm{~Hz}, 3 \mathrm{H}), 1.07(\mathrm{~s}, 3 \mathrm{H}), 0.75(\mathrm{~d}, J=6.6 \mathrm{~Hz}, 3 \mathrm{H}) ;{ }^{13} \mathrm{C}$ NMR $\left(100 \mathrm{MHz}, \mathrm{CDCl}_{3}\right) \delta$ $166.86,165.11,159.13,132.07,130.40,114.24,105.63,55.36,54.11,49.00,29.54,28.79$, 28.24, 21.84, 21.47; MS m/z (relative intensity) $307\left[(\mathrm{M}+1)^{+}, 3\right], 306\left(\mathrm{M}^{+}, 15\right), 220(30), 204$ $\left[\left(\mathrm{M}-\mathrm{CO}_{2}-\mathrm{C}_{3} \mathrm{H}_{6} \mathrm{O}\right)^{+}, 1\right], 163$ (55), 161 (100), 70 (21), 61 (24); HRMS (EI) m/z calcd for $\mathrm{C}_{17} \mathrm{H}_{22} \mathrm{O}_{5}$ $\left(\mathrm{M}^{+}\right)$306.1467, found 306.1473.

5-[Cyclohexyl(4-methoxyphenyl)methyl]-2,2-dimethyl-1,3-dioxane-4,6-dione 1c. (Table 1, entry 3). Yield 86\% (297 mg); White solid, m.p. 120-122 ${ }^{\circ} \mathrm{C}$; IR (KBr, neat) $v_{\max } 2928,2856$, 2358, 2339, 1738, 1712, 1611, 1513, 1252, 1181, 1115, 1031; ${ }^{1} \mathrm{H}$ NMR (400 MHz, $\left.\mathrm{CDCl}_{3}\right) \delta$ 7.18-7.16 (m, 2H), 6.82-6.80 (m, 2H), $3.89(\mathrm{~d}, J=3.1 \mathrm{~Hz}, 1 \mathrm{H}), 3.76(\mathrm{~s}, 3 \mathrm{H}), 3.33(\mathrm{dd}, J=11.2$, $3.0 \mathrm{~Hz}, 1 \mathrm{H}), 2.39-2.30(\mathrm{~m}, 1 \mathrm{H}), 2.06-2.03(\mathrm{~m}, 1 \mathrm{H}), 1.85-1.81(\mathrm{~m}, 1 \mathrm{H}), 1.68-1.45(\mathrm{~m}, 2 \mathrm{H}), 1.56$ $(\mathrm{s}, 3 \mathrm{H}), 1.45-1.36(\mathrm{~m}, 2 \mathrm{H}), 1.20-1.04(\mathrm{~m}, 3 \mathrm{H}), 1.10(\mathrm{~s}, 3 \mathrm{H}), 0.76-0.66(\mathrm{~m}, 1 \mathrm{H}) ;{ }^{13} \mathrm{C}$ NMR $(100$ $\left.\mathrm{MHz}_{,} \mathrm{CDCl}_{3}\right) \delta 166.91,165.10,159.07,131.94,130.53,114.21,105.52,55.36,52.52,48.10$, $38.26,32.14,31.46,28.69,28.24,26.44,26.28,26.16$; MS m/z (relative intensity) $346\left(\mathrm{M}^{+}, 16\right)$ 260 (39), 203 (36), 178 (25), 161 (100), 137 (23), 121 (69), 85 (24), 69 (21), 57 (42), 55 (24); HRMS (EI) $m / z$ calcd for $\mathrm{C}_{20} \mathrm{H}_{26} \mathrm{O}_{5}\left(\mathrm{M}^{+}\right) 346.1780$, found 346.1785 .

5-[1-(4-Methoxyphenyl)-2,2-dimethylpropyl]-2,2-dimethyl-1,3-dioxane-4,6-dione

1d. (Table 1, entry 4) Yield 96\% (307 mg); White solid, m.p. 143-144 ${ }^{\circ} \mathrm{C}$; IR (KBr, neat) $v_{\max }$ 2973, 1749, 1708, 1614, 1514, 1397, 1274, 1115, 1033, 827, 772, 703, 568, 546; ${ }^{1} \mathrm{H}$ NMR $(400$ $\left.\mathrm{MHz}, \mathrm{CDCl}_{3}\right) \delta$ 7.23-7.20 (m, 2H), 6.81-6.78 (m, 2H), $3.97(\mathrm{~d}, J=2.2 \mathrm{~Hz}, 1 \mathrm{H}), 3.77(\mathrm{~s}, 3 \mathrm{H})$, $3.53(\mathrm{~d}, J=2.2 \mathrm{~Hz}, 1 \mathrm{H}), 1.62(\mathrm{~s}, 3 \mathrm{H}), 1.13(\mathrm{~s}, 9 \mathrm{H}), 1.10(\mathrm{~s}, 3 \mathrm{H}) ;{ }^{13} \mathrm{C} \mathrm{NMR}\left(100 \mathrm{MHz}, \mathrm{CDCl}_{3}\right) \delta$ $168.09,165.61,159.18,132.24,130.53,113.79,105.51,57.89,55.37,48.87,35.40,29.55$, 28.92, 28.30; MS m/z (relative intensity) $321\left[(\mathrm{M}+1)^{+}, 1\right], 320\left(\mathrm{M}^{+}, 8\right), 234(24), 178(74), 161$ (100), 57 (28); HRMS (EI) $\mathrm{m} / \mathrm{z}$ calcd for $\mathrm{C}_{18} \mathrm{H}_{24} \mathrm{O}_{5}\left(\mathrm{M}^{+}\right) 320.1624$, found 320.1621 . 
5-[1-(4-Hydroxyphenyl)-propyl]-2,2-dimethyl-1,3-dioxane-4,6-dione 2a. (Table 1, entry 5) Yield 90\% (222 mg); White solid, m.p. 124-125 ${ }^{\circ} \mathrm{C} ;{ }^{1} \mathrm{H}$ NMR (400 MHz, $\left.\mathrm{CDCl}_{3}\right) \delta 7.13(\mathrm{~d}, J=$ $8.4 \mathrm{~Hz}, 2 \mathrm{H}), 6.76(\mathrm{~d}, J=8.4 \mathrm{~Hz}, 2 \mathrm{H}), 5.99(\mathrm{br}, 1 \mathrm{H}), 3.70(\mathrm{~d}, J=3.0 \mathrm{~Hz}, 1 \mathrm{H}), 3.61-3.56(\mathrm{~m}, 1 \mathrm{H})$, $2.30-2.18(\mathrm{~m}, 1 \mathrm{H}), 2.12-2.01(\mathrm{~m}, 1 \mathrm{H}), 1.63(\mathrm{~s}, 3 \mathrm{H}), 1.17(\mathrm{~s}, 3 \mathrm{H}), 0.92(\mathrm{t}, J=7.3 \mathrm{~Hz}, 3 \mathrm{H}) ;{ }^{13} \mathrm{C}$ NMR $\left(100 \mathrm{MHz}, \mathrm{CDCl}_{3}\right) \delta 166.68,165.28,155.55,131.30,130.35,115.75,105.92,51.22$, 47.81, 28.64, 28.23, 26.16, 12.73; MS m/z (relative intensity) $279\left[(\mathrm{M}+1)^{+}, 2\right], 278\left(\mathrm{M}^{+}, 12\right)$, $220\left[\left(\mathrm{M}-\mathrm{C}_{3} \mathrm{H}_{6} \mathrm{O}\right)^{+}, 6\right], 192(72), 176\left[\left(\mathrm{M}-\mathrm{CO}_{2}-\mathrm{C}_{3} \mathrm{H}_{6} \mathrm{O}\right)^{+}, 1\right], 174$ (26), 147 (82), 135 (100), 107 (38); HRMS (EI) $\mathrm{m} / \mathrm{z}$ calcd for $\mathrm{C}_{15} \mathrm{H}_{18} \mathrm{O}_{5}\left(\mathrm{M}^{+}\right)$278.1154, found 278.1153.

5-[1-(4-Hydroxyphenyl)-2-methylpropyl]-2,2-dimethyl-1,3-dioxane-4,6-dione 2b. (Table 1, entry 6) Yield $80 \%$ (233 mg); White solid, m.p. $109-111{ }^{\circ} \mathrm{C} ;{ }^{1} \mathrm{H}$ NMR (400 $\left.\mathrm{MHz}, \mathrm{CDCl}_{3}\right) \delta$ $7.10(\mathrm{~d}, J=8.5 \mathrm{~Hz}, 2 \mathrm{H}), 6.75(\mathrm{~d}, J=8.6 \mathrm{~Hz}, 2 \mathrm{H}), 3.87(\mathrm{~d}, J=3.2 \mathrm{~Hz}, 1 \mathrm{H}), 3.23(\mathrm{dd}, J=11.4$, $3.2 \mathrm{~Hz}, 1 \mathrm{H}), 2.74-2.68(\mathrm{~m}, 1 \mathrm{H}), 1.61(\mathrm{~s}, 3 \mathrm{H}), 1.19(\mathrm{~d}, J=6.5 \mathrm{~Hz}, 3 \mathrm{H}), 1.08(\mathrm{~s}, 3 \mathrm{H}), 0.75(\mathrm{~d}, J=$ $6.6 \mathrm{~Hz}, 3 \mathrm{H}) ;{ }^{13} \mathrm{C}$ NMR $\left(100 \mathrm{MHz}, \mathrm{CDCl}_{3}\right) \delta 167.16,165.28,155.42,131.95,130.58,115.82$, $105.89,54.25,48.99,29.57,28.87,28.28,21.84,21.50 ; \mathrm{MS} \mathrm{m} / \mathrm{z}$ (relative intensity) $293\left[(\mathrm{M}+1)^{+}\right.$, 1], $292\left(\mathrm{M}^{+}, 8\right), 149$ (80), 148 (90), 147 (78), 133 (53), 120 (33), 119 (21), 107 (100), 105 (24), 91 (46), 77 (43), 65 (22), 51 (26); HRMS (ESI) m/z calcd for $\mathrm{C}_{16} \mathrm{H}_{19} \mathrm{O}_{5}$ (M) 291.1232, found 291.1230 .

5-[Cyclohexyl(4-hydroxyphenyl)methyl]-2,2-dimethyl-1,3-dioxane-4,6-dione 2c. (Table 1, entry 7). Yield 95\% (315 mg); Yellow solid, m.p. 159-161 ${ }^{\circ} \mathrm{C} ;{ }^{1} \mathrm{H}$ NMR (400 $\left.\mathrm{MHz}, \mathrm{CDCl}_{3}\right) \delta$ 7.27-7.23 (m, 2H), 6.99-6.95 (m, 2H), $3.88(\mathrm{~d}, J=3.0 \mathrm{~Hz}, 1 \mathrm{H}), 3.39(\mathrm{dd}, J=11.3,3.0 \mathrm{~Hz}, 1 \mathrm{H})$, 2.39-2.32 (m, 1H), 2.03-1.99 (m, 1H), 1.85-1.82 (m, 1H), 1.69-1.60 (m, 2H), $1.63(\mathrm{~s}, 3 \mathrm{H}), 1.44-$ $1.36(\mathrm{~m}, 2 \mathrm{H}), 1.23-1.03(\mathrm{~m}, 3 \mathrm{H}), 1.20(\mathrm{~s}, 3 \mathrm{H}), 0.77-0.68(\mathrm{~m}, 1 \mathrm{H}) ;{ }^{13} \mathrm{C} \mathrm{NMR}\left(100 \mathrm{MHz}, \mathrm{CDCl}_{3}\right)$ $\delta 166.59,165.00,163.53,161.08,135.75,135.72,131.23,131.15,115.83,115.62,105.50$, $52.29,48.08,38.32,32.18,31.41,28.57,28.25,26.40,26.26,26.13 ;$ MS m/z (relative intensity) $332\left(\mathrm{M}^{+}, 1\right), 311$ (1), 210 (3), 191 (6), 154 (10), 109 (20), 95 (24), 55 (100), 43 (72), 29 (17); HRMS (ESI) $\mathrm{m} / \mathrm{z}$ calcd for $\mathrm{C}_{19} \mathrm{H}_{23} \mathrm{O}_{5}\left(\mathrm{M}^{-}\right) 331.1545$, found 331.1536 .

(Table 1, entry 8). Yield 87\% (266 mg); Yellow solid, m.p. 145-148 ${ }^{\circ} \mathrm{C}$; ${ }^{1} \mathrm{H}$ NMR (400 MHz, $\left.\mathrm{CDCl}_{3}\right) \delta 7.17(\mathrm{~d}, J=8.6 \mathrm{~Hz}, 2 \mathrm{H}), 6.73(\mathrm{~d}, J=8.6 \mathrm{~Hz}, 2 \mathrm{H}), 4.93(\mathrm{br}, 1 \mathrm{H}), 3.96(\mathrm{~d}, J=2.1 \mathrm{~Hz}$, $1 \mathrm{H}), 3.52(\mathrm{~d}, J=2.1 \mathrm{~Hz}, 1 \mathrm{H}), 1.62(\mathrm{~s}, 3 \mathrm{H}), 1.13(\mathrm{~s}, 9 \mathrm{H}), 1.11(\mathrm{~s}, 3 \mathrm{H}) ;{ }^{13} \mathrm{C}$ NMR $(100 \mathrm{MHz}$, $\left.\mathrm{CDCl}_{3}\right) \delta 168.14,165.68,155.28,132.47,130.68,115.33,105.61,57.93,48.89,35.41,29.57$, 28.96, 28.35; MS m/z (relative intensity) $307\left[(\mathrm{M}+1)^{+}, 7\right], 306\left(\mathrm{M}^{+}, 1\right), 252$ (5), 231 (3), 163 (100), 154 (55), 136 (40), 107 (28), 57 (50), 55 (31), 43 (30); HRMS (EI) m/z calcd for $\mathrm{C}_{17} \mathrm{H}_{22} \mathrm{O}_{5}\left(\mathrm{M}^{+}\right)$306.1467, found 306.1481.

5-\{1-[4-(Dimethylamino)phenyl]propyl\}-2,2-dimethyl-1,3-dioxane-4,6-dione 3a. (Table 1, entry 9). Yield 89\% (271 mg); Reddish orange oil; ${ }^{1} \mathrm{H}$ NMR (400 MHz, $\left.\mathrm{CDCl}_{3}\right) \delta 7.17$ (d, $J=$ $8.7 \mathrm{~Hz}, 2 \mathrm{H}), 6.66(\mathrm{~d}, J=8.5 \mathrm{~Hz}, 2 \mathrm{H}), 3.66(\mathrm{~d}, J=3.1 \mathrm{~Hz}, 1 \mathrm{H}), 3.58-3.53(\mathrm{~m}, 1 \mathrm{H}), 2.91(\mathrm{~s}, 3 \mathrm{H})$, $2.30-2.18(\mathrm{~m}, 1 \mathrm{H}), 2.11-2.01(\mathrm{~m}, 1 \mathrm{H}), 1.62(\mathrm{~s}, 3 \mathrm{H}), 1.13(\mathrm{~s}, .3 \mathrm{H}), 0.93(\mathrm{t}, J=7.3 \mathrm{~Hz}, 3 \mathrm{H}) ;{ }^{13} \mathrm{C}$ NMR $\left(100 \mathrm{MHz}, \mathrm{CDCl}_{3}\right) \delta 166.52,165.18,150.12,129.82,127.40,112.96,105.68,51.39$, $47.84,40.83,28.74,28.30,26.19,12.85 ; \mathrm{MS} \mathrm{m} / \mathrm{z}$ (relative intensity) $305\left(\mathrm{M}^{+}, 3\right), 247$ [(M- 
$\left.\left.\mathrm{C}_{3} \mathrm{H}_{6} \mathrm{O}\right)^{+}, 1\right], 203$ [( $\left.\left(\mathrm{M}-\mathrm{CO}_{2}-\mathrm{C}_{3} \mathrm{H}_{6} \mathrm{O}\right)^{+}, 11\right], 202$ (36), 174 (40), 162 (100). 149 (20), 147 (35), 146 (33), 134 (57); HRMS (EI) $\mathrm{m} / \mathrm{z}$ calcd for $\mathrm{C}_{17} \mathrm{H}_{23} \mathrm{NO}_{4}\left(\mathrm{M}^{+}\right) 305.1627$, found 305.1633 .

5-\{1-[4-(Dimethylamino)phenyl]-2-methylpropyl\}-2,2-dimethyl-1,3-dioxane-4,6-dione $3 \mathrm{~b}$. (Table 1, entry 10). Yield $84 \%$ (268 mg); Reddish orange solid, m.p. 124-125 ${ }^{\circ} \mathrm{C}$; ${ }^{1} \mathrm{H}$ NMR $\left(400 \mathrm{MHz}, \mathrm{CDCl}_{3}\right) \delta 7.09(\mathrm{~d}, J=8.6 \mathrm{~Hz}, 2 \mathrm{H}), 6.63(\mathrm{~d}, J=8.6 \mathrm{~Hz}, 2 \mathrm{H}), 3.86(\mathrm{~d}, J=3.1 \mathrm{~Hz}, 2 \mathrm{H})$, $3.19(\mathrm{dd}, J=11.3,3.1 \mathrm{~Hz}, 1 \mathrm{H}), 2.90(\mathrm{~s}, 6 \mathrm{H}), 2.77-2.64(\mathrm{~m}, 1 \mathrm{H}), 1.60(\mathrm{~s}, 3 \mathrm{H}), 1.19(\mathrm{~d}, J=6.5$ $\mathrm{Hz}, 3 \mathrm{H}), 1.03$ (s, 3H), 0.76 (d, $J=6.6 \mathrm{~Hz}, 3 \mathrm{H}) ;{ }^{13} \mathrm{C} \mathrm{NMR}\left(100 \mathrm{MHz}, \mathrm{CDCl}_{3}\right) \delta 167.13,165.27$, $150.15,129.98,113.01,105.74,54.28,49.14,40.78,29.53,28.92,28.28,21.90,21.59 ; \mathrm{MS} \mathrm{m} / \mathrm{z}$

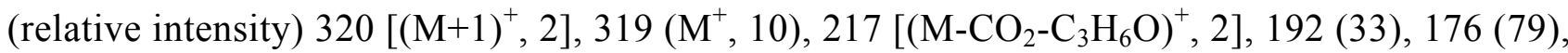
174 (100), 146 (34), 134 (25); HRMS (EI) m/z calcd for $\mathrm{C}_{18} \mathrm{H}_{25} \mathrm{NO}_{4}\left(\mathrm{M}^{+}\right)$319.1784, found 319.1781 .

5-\{Cyclohexyl[4-(dimethylamino)phenyl]methyl\}-2,2-dimethyl-1,3-dioxane-4,6-dione 3c. (Table 1, entry 11). Yield 90\% (323 mg); Orange solid, m.p. 130-131 ${ }^{\circ} \mathrm{C} ;{ }^{1} \mathrm{H}$ NMR (400 MHz, $\left.\mathrm{CDCl}_{3}\right) \delta 7.09(\mathrm{~d}, J=8.8 \mathrm{~Hz}, 2 \mathrm{H}), 6.63(\mathrm{~d}, J=8.7 \mathrm{~Hz}, 2 \mathrm{H}), 3.88(\mathrm{~d}, J=3.1 \mathrm{~Hz}, 1 \mathrm{H}), 3.28(\mathrm{dd}, J$

$=11.2,3.3 \mathrm{~Hz}, 1 \mathrm{H}), 2.89(\mathrm{~s}, 6 \mathrm{H}), 2.38-2.30(\mathrm{~m}, 1 \mathrm{H}), 2.06-2.03(\mathrm{~m}, 1 \mathrm{H}), 1.84-1.67(\mathrm{~m}, 1 \mathrm{H})$, 1.67-1.60 (m, 2H), $1.60(\mathrm{~s}, 3 \mathrm{H}), 1.50-1.35(\mathrm{~m}, 2 \mathrm{H}), 1.19-1.04(\mathrm{~m}, 3 \mathrm{H}), 1.06(\mathrm{~s}, 3 \mathrm{H}), 0.71-0.68$ $(\mathrm{m}, 1 \mathrm{H}) ;{ }^{13} \mathrm{C}$ NMR $\left(100 \mathrm{MHz}, \mathrm{CDCl}_{3}\right) \delta 167.16,165.25,150.08,130.08,127.63,112.91,105.59$, 52.66, 48.21, 40.72, 38.22, 32.14, 31.56, 28.78, 28.26, 26.50, 26.33, 26.22; MS $\mathrm{m} / \mathrm{z}$ (relative intensity) $360\left[(\mathrm{M}+1)^{+}, 54\right], 359\left(\mathrm{M}^{+}, 85\right), 302$ (21), 301 (6), 289 (5). 284 (3), 276 (6), 258 (8), 256 (16); HRMS (ESI) $\mathrm{m} / \mathrm{z}$ calcd for $\mathrm{C}_{21} \mathrm{H}_{28} \mathrm{NO}_{4}$ (M) 358.2018, found 359.2009.

\section{5-\{1-[4-(Dimethylamino)phenyl]-2,2-dimethylpropyl\}-2,2-dimethyl-1,3-dioxane-4,6-dione} 3d. (Table 1, entry 12). Yield 95\% (315 mg); Reddish orange solid, m.p. 135-136 ${ }^{\circ} \mathrm{C} ;{ }^{1} \mathrm{H}$ NMR $\left(400 \mathrm{MHz}, \mathrm{CDCl}_{3}\right) \delta 7.14(\mathrm{~d}, J=8.8 \mathrm{~Hz}, 2 \mathrm{H}), 6.62(\mathrm{~d}, J=8.7 \mathrm{~Hz}, 2 \mathrm{H}), 3.97(\mathrm{~d}, J=2.1 \mathrm{~Hz}, 1 \mathrm{H})$, $3.45(\mathrm{~d}, J=2.0 \mathrm{~Hz}, 1 \mathrm{H}), 2.90(\mathrm{~s}, 6 \mathrm{H}), 1.61(\mathrm{~s}, 3 \mathrm{H}), 1.13(\mathrm{~s}, 9 \mathrm{H}), 1.07(\mathrm{~s}, 3 \mathrm{H}) ;{ }^{13} \mathrm{C}$ NMR $(100$ $\left.\mathrm{MHz}, \mathrm{CDCl}_{3}\right) \delta 168.42,165.77,150.03,131.83,126.18,112.39,105.59,58.21,48.91,40.66$, 35.60, 29.57, 29.03, 28.27; MS m/z (relative intensity) $333\left(\mathrm{M}^{+}, 55\right), 307$ (2), 262 (2), 251 (4), 250 (23). 249 (23), 248 (15), 190 (99), 174 (13); HRMS (EI) m/z calcd for $\mathrm{C}_{19} \mathrm{H}_{27} \mathrm{NO}_{4}\left(\mathrm{M}^{+}\right.$) 333.1940 , found 333.1930 .

5-[1-(4-Fluorophenyl)-propyl]-2,2-dimethyl-1,3-dioxane-4,6-dione 4a. (Table 1, entry 13). Yield 56\% (156 mg); White solid, m.p. 69-70 ${ }^{\circ} \mathrm{C} ;{ }^{1} \mathrm{H}$ NMR (400 MHz, $\left.\mathrm{CDCl}_{3}\right) \delta$ 7.31-7.27 (m, $2 \mathrm{H}), 7.01-6.97(\mathrm{~m}, 2 \mathrm{H}), 3.69-3.63(\mathrm{~m}, 2 \mathrm{H}), 2.32-2.21(\mathrm{~m}, 1 \mathrm{H}), 2.12-2.01(\mathrm{~m}, 1 \mathrm{H}), 1.65(\mathrm{~s}, 3 \mathrm{H})$, $1.27(\mathrm{~s}, 3 \mathrm{H}), 0.93(\mathrm{t}, J=7.3 \mathrm{~Hz}, 3 \mathrm{H}) ;{ }^{13} \mathrm{C}$ NMR $\left(100 \mathrm{MHz}, \mathrm{CDCl}_{3}\right) \delta 165.93,164.86,162.41(\mathrm{~d}$, $J=245 \mathrm{~Hz}), 135.37(\mathrm{~d}, J=3 \mathrm{~Hz}), 130.96(\mathrm{~d}, J=8 \mathrm{~Hz}), 115.69$ (d, $J=21 \mathrm{~Hz}), 51.23,47.31$, 28.49, 28.31, 26.03, 12.76; MS m/z (relative intensity) $280\left(\mathrm{M}^{+}, 1\right), 222\left[\left(\mathrm{M}-\mathrm{CO}_{2}\right)^{+}, 9\right], 194$ (100), 178 [(M-CO $\left.\left.-\mathrm{CO}_{2} \mathrm{H}_{6} \mathrm{O}\right)^{+}, 3\right], 176$ (24), 149 (81), 137 (30), 109 (40); HRMS (CI) m/z calcd for $\mathrm{C}_{15} \mathrm{H}_{17} \mathrm{FO}_{4}\left(\mathrm{M}^{+}\right)$280.1111, found 280.1110 .

5-[1-(4-Fluorophenyl)-2-methylpropyl]-2,2-dimethyl-1,3-dioxane-4,6-dione 4b. (Table 1, entry 14). Yield 40\% (117 mg); White solid, m.p. 79-80 ${ }^{\circ} \mathrm{C} ;{ }^{1} \mathrm{H}$ NMR $\left(400 \mathrm{MHz}, \mathrm{CDCl}_{3}\right) \delta$ 7.27-7.23 (m, 2H), 7.00-6.96 (m, 2H), $3.86(\mathrm{~d}, J=3.2 \mathrm{~Hz} .1 \mathrm{H}), 3.30$ (dd, $J=11.4,3.1 \mathrm{~Hz}, 1 \mathrm{H})$, 2.77-2.71 (m, 1H), $1.63(\mathrm{~s}, 3 \mathrm{H}), 1.19(\mathrm{~d}, J=6.5 \mathrm{~Hz}, 3 \mathrm{H}), 1.16(\mathrm{~s}, 3 \mathrm{H}), 0.75(\mathrm{~d}, J=6.6 \mathrm{~Hz}, 3 \mathrm{H})$; ${ }^{13} \mathrm{C}$ NMR $\left(100 \mathrm{MHz}, \mathrm{CDCl}_{3}\right) \delta 166.56,165.00,162.36(\mathrm{~d}, J=245 \mathrm{~Hz}), 135.86(\mathrm{~d}, J=5 \mathrm{~Hz})$, 
$131.08(\mathrm{~d}, J=8 \mathrm{~Hz}), 115.78(\mathrm{~d}, J=21 \mathrm{~Hz}), 105.61,53.87,48.97,29.61,28.70,28.26,21.86$,

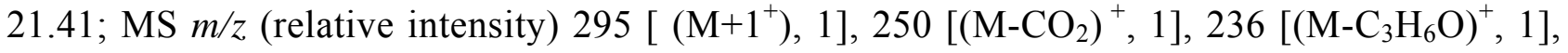
289 (3), 277 (1), 265 (3), 255 (12), 193 (5), 151 (91), 109 (40). HRMS (ESI) m/z calcd for $\mathrm{C}_{16} \mathrm{H}_{18} \mathrm{FO}_{4}\left(\mathrm{M}^{-}\right)$293.1189, found 293.1186.

5-[Cyclohexyl(4-fluorophenyl)methyl]-2,2-dimethyl-1,3-dioxane-4,6-dione 4c. (Table 1, entry 15). Yield 40\% (133 mg); White solid, m.p. 79-81 ${ }^{\circ} \mathrm{C} ;{ }^{1} \mathrm{H}$ NMR (400 MHz, $\left.\mathrm{CDCl}_{3}\right) \delta 7.25$ $(\mathrm{dd}, J=8.2,5.0 \mathrm{~Hz}, 2 \mathrm{H}), 6.97(\mathrm{t}, J=8.7 \mathrm{~Hz}, 2 \mathrm{H}), 3.88(\mathrm{~d}, J=3.0 \mathrm{~Hz}, 1 \mathrm{H}), 3.39(\mathrm{dd}, J=11.3$, $3.0 \mathrm{~Hz}, 1 \mathrm{H}), 2.41-2.32(\mathrm{~m}, 1 \mathrm{H}), 2.01(\mathrm{~d}, J=12.1 \mathrm{~Hz}, 1 \mathrm{H}), 1.83(\mathrm{~d}, J=13.3 \mathrm{~Hz}, 1 \mathrm{H}), 1.69-1.56$ $(\mathrm{m}, 2 \mathrm{H}), 1.63(\mathrm{~s}, 3 \mathrm{H}), 1.45-1.36(\mathrm{~m}, 2 \mathrm{H}), 1.20(\mathrm{~s}, 3 \mathrm{H}), 1.20-1.04(\mathrm{~m}, 3 \mathrm{H}), 0.77-0.67(\mathrm{~m}, 1 \mathrm{H})$;

${ }^{13} \mathrm{C}$ NMR $\left(100 \mathrm{MHz}, \mathrm{CDCl}_{3}\right) \delta 166.60,165.02,163.32(\mathrm{~d}, J=245 \mathrm{~Hz}), 135.76(\mathrm{~d}, J=3 \mathrm{~Hz})$, $131.20(\mathrm{~d}, J=8 \mathrm{~Hz}), 115.75(\mathrm{~d}, J=21 \mathrm{~Hz}), 52.30,48.09,38.34,32.19,31.43,28.59,26.41$,

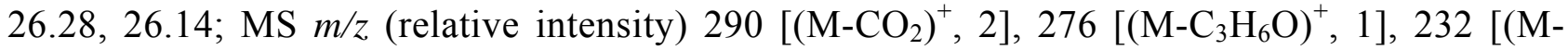
$\mathrm{CO}_{2}-\mathrm{C}_{3} \mathrm{H}_{6} \mathrm{O}$ )+, 2], 307 (9), 295 (8), 231 (4), 191 (61), 154 (57), 136 (45), 109 (93).

HRMS (ESI) $\mathrm{m} / \mathrm{z}$ calcd for $\mathrm{C}_{19} \mathrm{H}_{22} \mathrm{FO}_{4}\left(\mathrm{M}^{-}\right)$333.1502, found 333.1498.

5-[1-(4-Fluorophenyl)-2,2-dimethylpropyl]-2,2-dimethyl-1,3-dioxane-4,6-dione 4d. (Table 1, entry 16). Yield $62 \%$ (191 mg); White solid, m.p. 82-83 ${ }^{\circ} \mathrm{C} ;{ }^{1} \mathrm{H}$ NMR $\left(400 \mathrm{MHz}, \mathrm{CDCl}_{3}\right) \delta$ 7.32-7.29 (m, 2H), 6.99-6.65 (m, 2H), $3.96(\mathrm{~d}, J=2.2 \mathrm{~Hz}, 1 \mathrm{H}), 3.62(\mathrm{~d}, J=2.2 \mathrm{~Hz}, 1 \mathrm{H}), 1.65$ (s, $3 \mathrm{H}), 1.19(\mathrm{~s}, 3 \mathrm{H}), 1.13(\mathrm{~s}, 9 \mathrm{H}) ;{ }^{13} \mathrm{C} \mathrm{NMR}\left(100 \mathrm{MHz}, \mathrm{CDCl}_{3}\right) \delta 167.65,165.41,162.45(\mathrm{~d}, J=$ $246 \mathrm{~Hz}), 134.30$ (d, $J=3 \mathrm{~Hz}), 132.85$ (d, $J=8 \mathrm{~Hz}), 115.33$ (d, $J=21 \mathrm{~Hz}), 105.44,57.37,48.87$, 35.23, 29.57, 28.74, 28.33; MS m/z (relative intensity) $309\left[(\mathrm{M}+1)^{+}, 5\right], 308\left(\mathrm{M}^{+}, 1\right), 307$ (4), 251 (18), 233 (32), 212 (15), 166 (21), 165 (90), 109 (35); HRMS (EI) m/z calcd for $\mathrm{C}_{17} \mathrm{H}_{21} \mathrm{FO}_{4}$ $\left(\mathrm{M}^{+}\right) 308.1424$, found 308.1413 .

5-[1-(2-Furyl)propyl]-2,2-dimethyl-1,3-dioxane-4,6-dione 5a. (Table 1, entry 17). Yield 77\% (194 mg); Colorless oil; ${ }^{1} \mathrm{H}$ NMR (400 MHz, $\left.\mathrm{CDCl}_{3}\right) \delta 7.31$ (d, $\left.J=0.8 \mathrm{~Hz}, 1 \mathrm{H}\right), 6.31-6.29$ $(\mathrm{m}, 1 \mathrm{H}), 6.20(\mathrm{~d}, J=3.2 \mathrm{~Hz}, 1 \mathrm{H}), 3.82-3.77(\mathrm{~m}, 1 \mathrm{H}), 7.76(\mathrm{~d}, J=2.9 \mathrm{~Hz}, 1 \mathrm{H}), 2.28-2.17(\mathrm{~m}$, $1 \mathrm{H}), 1.99-1.88(\mathrm{~m}, 1 \mathrm{H}), 1.73(\mathrm{~s}, 3 \mathrm{H}), 1.54(\mathrm{~s}, 3 \mathrm{H}), 1.01(\mathrm{t}, J=7.4 \mathrm{~Hz}, 3 \mathrm{H}) ;{ }^{13} \mathrm{C} \mathrm{NMR}(100 \mathrm{MHz}$, $\left.\mathrm{CDCl}_{3}\right) \delta 165.34,164.55,153.98,141.64,110.69,107.61,105.35,48.93,41.05,28.30,28.04$,

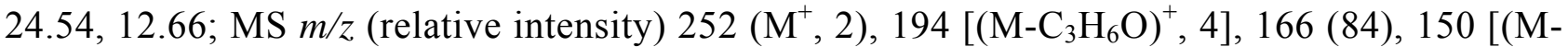
$\left.\mathrm{CO}_{2}-\mathrm{C}_{3} \mathrm{H}_{6} \mathrm{O}\right)^{+}, 3$ ], 121 (72), 120 (20), 109 (28), 107 (25), 93 (33), 81 (40), 79 (27), 65 (100); HRMS (EI) $\mathrm{m} / \mathrm{z}$ calcd for $\mathrm{C}_{13} \mathrm{H}_{16} \mathrm{O}_{5}\left(\mathrm{M}^{+}\right)$252.0998, found 252.0998 .

5-[1-(2-Furyl)-2-methylpropyl]-2,2-dimethyl-1,3-dioxane-4,6-dione 5b. (Table 1, entry 18). Yield 72\% (191 mg); Yellow solid, m.p. 93-94 ${ }^{\circ} \mathrm{C} ;{ }^{1} \mathrm{H}$ NMR (400 MHz, $\left.\mathrm{CDCl}_{3}\right) \delta 7.31$ (d, $J=$ $1.0 \mathrm{~Hz}, 1 \mathrm{H}), 6.29-6.27(\mathrm{~m}, 1 \mathrm{H}), 6.19(\mathrm{~d}, J=3.1 \mathrm{~Hz}, 1 \mathrm{H}), 3.82(\mathrm{~d}, J=3.1 \mathrm{~Hz}, 1 \mathrm{H}), 2.75-2.65(\mathrm{~m}$, $1 \mathrm{H}), 1.69(\mathrm{~s}, 3 \mathrm{H}), 1.37(\mathrm{~s}, 3 \mathrm{H}), 1.13(\mathrm{~d}, J=6.6 \mathrm{~Hz}, 3 \mathrm{H}), 0.85(\mathrm{~d}, J=6.6 \mathrm{~Hz}, 3 \mathrm{H}) ;{ }^{13} \mathrm{C}$ NMR $\left(100 \mathrm{MHz}, \mathrm{CDCl}_{3}\right) \delta 166.34,164.53,141.86,110.58,108.71,105.52,47.78,47.39,29.18,28.47$,

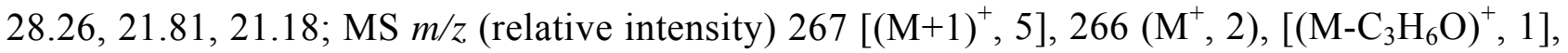
158 (1), 226 (10), 209 (11), 181 (8), 180 (37), 154 (16), 136 (13), 123 (100), 121 (27), 57 (10); HRMS (ESI) $\mathrm{m} / \mathrm{z}$ calcd for $\mathrm{C}_{14} \mathrm{H}_{17} \mathrm{O}_{5}\left(\mathrm{M}^{-}\right)$265.1076, found 265.1076.

5-[Cyclohexyl(2-furyl)methyl]-2,2-dimethyl-1,3-dioxane-4,6-dione 5c. (Table 1, entry 19). Yield 87\% (266 mg); White solid, m.p. 122-123 ${ }^{\circ} \mathrm{C} ;{ }^{1} \mathrm{H}$ NMR (400 MHz, $\left.\mathrm{CDCl}_{3}\right) \delta 7.31(\mathrm{~s}, 1 \mathrm{H})$, $6.26(\mathrm{~m}, 1 \mathrm{H}), 6.18(\mathrm{~d}, J=2.9 \mathrm{~Hz}, 1 \mathrm{H}), 3.83(\mathrm{~d}, J=1.5 \mathrm{~Hz}, 1 \mathrm{H}), 3.54(\mathrm{dd}, J=11.6,2.8 \mathrm{~Hz}, 1 \mathrm{H})$, 
1.94-1.91 (m, 1H), 1.82-1.78 (m, 1H), $1.68(\mathrm{~s}, 3 \mathrm{H}), 1.68-1.60(\mathrm{~m}, 2 \mathrm{H}), 1.52-1.49(\mathrm{~m}, 1 \mathrm{H}), 1.39$ $(\mathrm{s}, 3 \mathrm{H}), 1.42-1.33(\mathrm{~m}, 1 \mathrm{H}), 1.28-1.11(\mathrm{~m}, 1 \mathrm{H}), 1.07-1.00(\mathrm{~m}, 1 \mathrm{H}), 0.86-0.79(\mathrm{~m}, 1 \mathrm{H}) ;{ }^{13} \mathrm{C}$ NMR $\left(100 \mathrm{MHz}, \mathrm{CDCl}_{3}\right) \delta 166.35,164.52,153.75,141.86,110.57,108.78,105.43,46.62,46.38$, 37.91, 32.26, 31.33, 28.40, 28.29, 26.44, 26.26, 26.12; MS m/z (relative intensity) $306\left(\mathrm{M}^{+}, 1\right)$, $248\left[\left(\mathrm{M}-\mathrm{C}_{3} \mathrm{H}_{6} \mathrm{O}\right)^{+}, 3\right], 204\left[\left(\mathrm{M}-\mathrm{CO}_{2}-\mathrm{C}_{3} \mathrm{H}_{6} \mathrm{O}\right)^{+}, 7\right], 138$ (52), 121 (100), 81 (28), 55 (23); HRMS (EI) $\mathrm{m} / \mathrm{z}$ calcd for $\mathrm{C}_{17} \mathrm{H}_{22} \mathrm{O}_{5}\left(\mathrm{M}^{+}\right) 306.1467$, found 306.1468 .

5-[1-(2-Furyl)-2,2-dimethylpropyl]-2,2-dimethyl-1,3-dioxane-4,6-dione 5d. (Table 1, entry 20). Yield 91\% (254 mg); Yellow solid, m.p. 95-96 ${ }^{\circ} \mathrm{C} ;{ }^{1} \mathrm{H} \mathrm{NMR}\left(400 \mathrm{MHz}, \mathrm{CDCl}_{3}\right) \delta 7.30(\mathrm{~s}$, $1 \mathrm{H}), 6.30-6.28(\mathrm{~m}, 1 \mathrm{H}), 6.23(\mathrm{~d}, J=3.0 \mathrm{~Hz}, 1 \mathrm{H}), 3.88(\mathrm{~d}, J=1.6 \mathrm{~Hz}, 1 \mathrm{H}), 3.73(\mathrm{~d}, J=1.3 \mathrm{~Hz}$, $1 \mathrm{H}), 1.68(\mathrm{~s}, 3 \mathrm{H}), 1.31(\mathrm{~s}, 3 \mathrm{H}), 1.12(\mathrm{~s}, 9 \mathrm{H}) ;{ }^{13} \mathrm{C} \mathrm{NMR}\left(100 \mathrm{MHz}, \mathrm{CDCl}_{3}\right) \delta 167.76,164.51$, $152.40,141.46,110.78,110.04,105.42,51.16,47.26,35.52,29.04,28.49,28.31 ; \mathrm{MS} \mathrm{m} / \mathrm{z}$

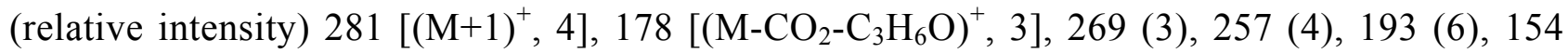
(17), 137 (24), 111 (14), 95 (33), 83 (41), 69 (60), 57 (100). HRMS (ESI) m/z calcd for $\mathrm{C}_{15} \mathrm{H}_{19} \mathrm{O}_{5}\left(\mathrm{M}^{-}\right) 279.1232$, found 279.1960.

5-(1-Ethyl-3-methylbutyl)-2,2-dimethyl-1,3-dioxane-4,6-dione 6a. (Table 1, entry 21). Yield 84\% (203 mg); Colorless oil; ${ }^{1} \mathrm{H}$ NMR (400 MHz, $\left.\mathrm{CDCl}_{3}\right) \delta 3.47$ (d, J=3.5 Hz, 1H), 2.53-2.45 $(\mathrm{m}, 1 \mathrm{H}), 1.75(\mathrm{~s}, 6 \mathrm{H}), 1.66-1.53(\mathrm{~m}, 3 \mathrm{H}), 1.46-1.32(\mathrm{~m}, 2 \mathrm{H}), 0.96-0.89(\mathrm{~m}, 9 \mathrm{H}) ;{ }^{13} \mathrm{C}$ NMR $(100$ $\left.\mathrm{MHz}, \mathrm{CDCl}_{3}\right) \delta 166.03,165.78,104.83,47.88,41.08,39.43,28.33,27.94,25.82,24.63,22.92$, 22.66, 12.49; MS m/z (relative intensity) 203 (3), 185 (3), 171 (3), 154 (11), 136 (10), 123 (8), 111 (10), 97 (50), 83 (39), 57 (100), 43 (69). HRMS (ESI) m/z calcd for $\mathrm{C}_{13} \mathrm{H}_{21} \mathrm{O}_{4}\left(\mathrm{M}^{-}\right.$) 241.1440, found 241.1437 .

5-(1-Isopropyl-3-methylbutyl)-2,2-dimethyl-1,3-dioxane-4,6-dione 6b. (Table 1, entry 22). Yield 80\% (204 mg); Colorless oil; ${ }^{1} \mathrm{H}$ NMR (400 MHz, $\left.\mathrm{CDCl}_{3}\right) \delta 3.42(\mathrm{~d}, J=1.9 \mathrm{~Hz}, 1 \mathrm{H})$, 2.40-2.34 (m, 1H), 1.91-1.84 (m, 1H), $1.74(\mathrm{~s}, 6 \mathrm{H}), 1.65-1.51(\mathrm{~m}, 2 \mathrm{H}), 1.41-1.32(\mathrm{~m}, 1 \mathrm{H}), 0.97-$ $0.89(\mathrm{~m}, 9 \mathrm{H}), 0.85(\mathrm{~d}, J=6.8 \mathrm{~Hz}, 3 \mathrm{H}) ;{ }^{13} \mathrm{C} \mathrm{NMR}\left(100 \mathrm{MHz}, \mathrm{CDCl}_{3}\right) \delta 166.79,165.71,104.83$, $68.12,46.90,44.13,40.15,30.32,28.24,26.62,23.78,21.90,21.54,21.22 ; \mathrm{MS} \mathrm{m} / \mathrm{z}$ (relative

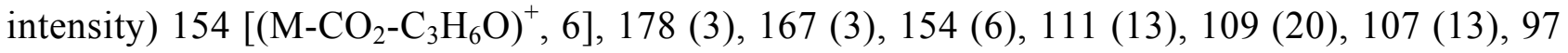
(28), 69 (68), 57 (73), 55 (100), 43 (61). HRMS (FAB) $\mathrm{m} / \mathrm{z}$ calcd for $\mathrm{C}_{14} \mathrm{H}_{25} \mathrm{O}_{4}\left(\mathrm{M}^{+}\right)$257.1753, found 257.1747 .

5-(1-Cyclohexyl-3-methylbutyl)-2,2-dimethyl--1,3-dioxane-4,6-dione 6c. (Table 1, entry 23). Yield 92\% (272 mg); Colorless solid, m.p. 84-85 ${ }^{\circ} \mathrm{C} ;{ }^{1} \mathrm{H}$ NMR (400 MHz, $\left.\mathrm{CDCl}_{3}\right) \delta 3.42(\mathrm{~s}, 1 \mathrm{H})$, 2.40-2.37 (m, 1H), $1.91(\mathrm{~d}, J=12.0 \mathrm{~Hz}, 1 \mathrm{H}), 1.80-1.70(\mathrm{~m}, 7 \mathrm{H}), 1.64-1.59(\mathrm{~m}, 2 \mathrm{H}), 1.57-1.52$ $(\mathrm{m}, 2 \mathrm{H}), 1.45-1.39(\mathrm{~m}, 2 \mathrm{H}), 1.25-0.89(\mathrm{~m}, 5 \mathrm{H}), 0.94(\mathrm{~d}, J=6.0 \mathrm{~Hz}, 3 \mathrm{H}), 0.90(\mathrm{~d}, J=6.0 \mathrm{~Hz}$, $3 \mathrm{H}) ;{ }^{13} \mathrm{C} \mathrm{NMR}\left(100 \mathrm{MHz}, \mathrm{CDCl}_{3}\right) \delta 166.50,165.53,104.62,46.54,42.77,39.99,39.94,31.66$, $31.56,28.14,27.60,26.63,26.59,26.46,26.33,23.56,21.80 ; \mathrm{MS} \mathrm{m} / \mathrm{z}$ (relative intensity) 289 (4), 285 (6), 225 (11), 155 (12), 154 (39), 151 (28), 137 (30), 123 (12), 111 (18), 97 (49), 55 (100). HRMS (ESI) $\mathrm{m} / \mathrm{z}$ calcd for $\mathrm{C}_{17} \mathrm{H}_{27} \mathrm{O}_{4}\left(\mathrm{M}^{-}\right)$295.1909, found 295.1909.

5-(1-tert-Butyl-3-methylbutyl)-2,2-dimethyl-1,3-dioxane-4,6-dione 6d. (Table 1, entry 24).Yield 70\% (189 mg); Colorless oil; ${ }^{1} \mathrm{H} \mathrm{NMR}\left(400 \mathrm{MHz}, \mathrm{CDCl}_{3}\right) \delta 3.45(\mathrm{~d}, \mathrm{~J}=1.2 \mathrm{~Hz}, 1 \mathrm{H})$, $2.69(\mathrm{dd}, J=11.3,1.2 \mathrm{~Hz}, 1 \mathrm{H}), 1.88(\mathrm{t}, J=11.2 \mathrm{~Hz}, 1 \mathrm{H}), 1.75(\mathrm{~d}, J=7.7 \mathrm{~Hz}, 6 \mathrm{H}), 1.27-1.24(\mathrm{~m}$, $2 \mathrm{H}), 0.99$ (s, 9H), 0.93-0.83 (m, 6H); $\left.{ }^{13} \mathrm{C} \mathrm{NMR} \mathrm{(100} \mathrm{MHz,} \mathrm{CDCl}_{3}\right) \delta 167.37,165.91,104.59$, 
$47.74,45.49,36.66,34.19,29.03,28.13,28.02,26.56,24.34,21.70 ; \mathrm{MS} \mathrm{m} / \mathrm{z}$ (relative intensity) 177 (3), 149 (6), 137 (7), 123 (14), 109 (28), 97 (31), 95 (50), 81 (54), 69 (76), 57 (75), 55 (100), 43 (66). HRMS (ESI) $\mathrm{m} / \mathrm{z}$ calcd for $\mathrm{C}_{15} \mathrm{H}_{27} \mathrm{O}_{4}\left(\mathrm{M}^{+}\right)$271.1909, found 271.1907.

5-[Adamantan-1-yl(4-methoxyphenyl)methyl]-2,2-dimethyl-1,3-dioxane-4,6-dione

(Scheme 2). Yield 50\% (199 mg); White solid, m.p. 111-113 ${ }^{\circ} \mathrm{C}$; IR (KBr, neat) $v_{\max } 2909$, 2850, 1770, 1738, 1608, 1513, 1392, 1286, 1252, 1185, 1091, 1034, 1005, 944, 903, 864, 836, 786, 760, 663, 545; ${ }^{1} \mathrm{H}$ NMR $\left(400 \mathrm{MHz}, \mathrm{CDCl}_{3}\right) \delta 7.19(\mathrm{~d}, J=8.5 \mathrm{~Hz}, 2 \mathrm{H}), 6.80(\mathrm{~d}, J=8.6 \mathrm{~Hz}$, 2H), $4.02(\mathrm{~d}, J=1.3 \mathrm{~Hz}, 1 \mathrm{H}), 3.77(\mathrm{~s}, 3 \mathrm{H}), 3.32(\mathrm{~s}, 1 \mathrm{H}), 2.01(\mathrm{~s}, 3 \mathrm{H}), 1.79-1.56(\mathrm{~m}, 15 \mathrm{H}), 1.08$ (s, $3 \mathrm{H}) ;{ }^{13} \mathrm{C}$ NMR $\left(100 \mathrm{MHz}, \mathrm{CDCl}_{3}\right) \delta 168.38,165.71,159.16,132.58,129.73,113.74,105.47$, 59.57, 55.37, 47.55, 41.35, 36.90, 36.83, 29.05, 28.86, 28.35; MS m/z (relative intensity) 398

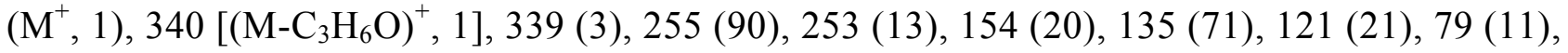
43 (10), 29 (3); HRMS (EI) $\mathrm{m} / \mathrm{z}$ calcd for $\mathrm{C}_{24} \mathrm{H}_{30} \mathrm{O}_{5}\left(\mathrm{M}^{+}\right)$398.2093, found 398.2102.

3-Ethyl-5,6-dimethoxyindan-1-one 9a. (Scheme 5) Yield 60\% (132 mg); Colorless solid, m.p. 90-91 ${ }^{\circ} \mathrm{C}$; IR (KBr, neat) $v_{\max } 2959,2929,2871,1695,1605,1588,1497,1465,1438,1416$, 1364, 1320, 1296, 1259, 1208, 1122, 1038, 1026, 981, 857, 845, 815, 719, 643, 544, 481; ${ }^{1} \mathrm{H}$ NMR (400 MHz, $\left.\mathrm{CDCl}_{3}\right) \delta 7.16(\mathrm{~s}, 1 \mathrm{H}), 6.90(\mathrm{~s}, 1 \mathrm{H}), 3.98(\mathrm{~s}, 3 \mathrm{H}), 3.91(\mathrm{~s}, 3 \mathrm{H}), 3.28-3.22(\mathrm{dd}, J$ $=18.9,7.2 \mathrm{~Hz}, 1 \mathrm{H}), 2.34(\mathrm{dd}, J=18.8,2.8 \mathrm{~Hz}, 1 \mathrm{H}), 2.02-1.90(\mathrm{~m}, 1 \mathrm{H}), 1.58-1.47(\mathrm{~m}, 1 \mathrm{H}), 0.97$ $(\mathrm{t}, J=7.3 \mathrm{~Hz}, 3 \mathrm{H}) ;{ }^{13} \mathrm{C}$ NMR $\left(100 \mathrm{MHz}, \mathrm{CDCl}_{3}\right) \delta 205.22,155.60,154.16,149.69,129.93$, 106.60, 104.15, 56.39, 56.27, 42.83, 39.47, 28.84, 11.69; MS m/z (relative intensity) 221 $\left[(\mathrm{M}+1)^{+}, 5\right], 220\left(\mathrm{M}^{+}, 32\right), 192(20), 191(100), 57$ (23); HRMS (EI) $\mathrm{m} / \mathrm{z}$ calcd for $\mathrm{C}_{13} \mathrm{H}_{16} \mathrm{O}_{3}\left(\mathrm{M}^{+}\right)$ 220.1099 , found 220.1095 .

3-Isopropyl-5,6-dimethoxyindan-1-one 9b. (Scheme 5). Yield 63\% (147 mg); Colorless solid, m.p. 133-134 ${ }^{\circ} \mathrm{C}$; IR (KBr, neat) $v_{\max } 3391,3070,3011,2964,2870,2841,2723,2664,1698$, 1593, 1503, 1466, 1443, 1420, 1367, 1300, 1130, 1047, 1004, 870, 831, 807, 728, 565, 543, 522, 482; ${ }^{1} \mathrm{H}$ NMR $\left(400 \mathrm{MHz}, \mathrm{CDCl}_{3}\right) \delta 7.16(\mathrm{~s}, 1 \mathrm{H}), 6.89(\mathrm{~s}, 1 \mathrm{H}), 3.98(\mathrm{~s}, 3 \mathrm{H}), 3.91(\mathrm{~s}, 3 \mathrm{H}), 3.34-$ 3.32 (m, 1H), 2.63 (dd, $J=19.0,7.4 \mathrm{~Hz}, 1 \mathrm{H}), 2.42$ (dd, $J=19.0,2.5 \mathrm{~Hz}, 1 \mathrm{H}), 2.27-2.20(\mathrm{~m}, 1 \mathrm{H})$, $1.03(\mathrm{~d}, J=6.8 \mathrm{H} \mathrm{z}, 3 \mathrm{H}), 0.66(\mathrm{~d}, J=6.8 \mathrm{~Hz}, 3 \mathrm{H}) ;{ }^{13} \mathrm{C} \mathrm{NMR}\left(100 \mathrm{MHz}, \mathrm{CDCl}_{3}\right) \delta 205.53,155.58$, 153.34, 149.70, 130.55, 106.81, 104.13, 56.41, 56.28, 44.03, 38.61, 31.50, 21.35, 16.50; MS m/z (relative intensity) $235\left[(\mathrm{M}+1)^{+}, 3\right], 234\left(\mathrm{M}^{+}, 20\right), 191$ (100); HRMS (EI) $\mathrm{m} / \mathrm{z}$ calcd for $\mathrm{C}_{14} \mathrm{H}_{18} \mathrm{O}_{3}\left(\mathrm{M}^{+}\right)$234.1256, found 234.1253.

\section{Acknowledgements}

Financial support of this work by the National Science Council of the Republic of China and Nation Taiwan Normal University (TOP 001) is gratefully acknowledged.

\section{References}

1. (a) Perlmutter, P. Conjugate Addition Reactions in Organic Synthesis; Pergamon: Oxford, UK, 1992. (b) Leonard, J.;Diez-Barra, F.; Merino, S. Eur. J. Org. Chem. 1998, 2051. 
2. (a) Dambacher, J.; Bergdahl, M. Org. Lett. 2003, 5, 3539. (b) Duguet, N.; Harrison-Marchand, A.; Maddaluno, J.; Tomioka, K. Org. Lett. 2006, 8, 5745. (c) Deng, K.; Bensari-Bouguerra, A.; Whetstone, J.; Cohen, T. J. Org. Chem. 2006, 71, 2360. (d) Sakai, T; Kawamoto, Y.; Tomioka, K. J. Org. Chem. 2006, 71, 4706. (e) Hodgson, D. M.; Fleming, M. J.; Stanway, S. J. J. Org. Chem. 2007, 72, 4763. (f) Doudouh, A.; Woltermann, C.; Gros, P. C. J. Org. Chem. 2007, 72, 4978. (g) Godenschwager, P. F.; Collum, D. B. J. Am. Chem. Soc. 2008, 130, 8726.

3. (a) Cahiez, G.; Alami, M. Tetrahedron Lett. 1989, 30, 3541. (b) Cahiez, G.; Alami, M. Tetrahedron Lett. 1989, 30, 7365. (c) Cahiez, G.; Alami, M. Tetrahedron Lett. 1990, 31, 7423. (d) Pearson, A. J.; Kim, J. B. Org. Lett. 2003, 5, 2457. (e) Kopp, F.; Knochel, P. Org. Lett. 2007, 9, 1639. (f) Fleming, F. F.; Gudipati, S.; Vu, V. A.; Mycka, R. J.; Knochel P. Org. Lett. 2007, 9, 4507. (g) Ding, F.; Zhang, Y.; Qu, B.; Li, G.; Farina, V.; Lu, B. Z.; Senanayake C. H. Org. Lett. 2008, 10, 1067. (h) Lu, Z.; Chai, G.; Zhang, X.; Ma, S. Org. Lett. 2008, 10, 3517. (i) Rauhut, C. B.; Melzig, L.; Knochel P. Org. Lett. 2008, 10, 3891.

4. (a) Petrier, C.; deSouza Barbosa, J. C.; Duppy, C.; Luche, J. L. J. Org. Chem. 1985, 50, 5761. (b) Petrier, C.; Duppy, C.; Luche, J. L. Tetrahedron Lett. 1986, 27, 3149. (c) Waston, R. A.; Kjonaas, R. A. Tetrahedron Lett. 1986, 27, 1437. (d) Kjonaas, R. A.; Vawter, E. J. J. Org. Chem. 1986, 51, 3993. (e) Tuckmantel, W.; Oshima, K.; Nozaki, H. Chem. Ber. 1986, 119, 1581. (f) Jubert, C.; Knochel, P. J. Org. Chem. 1992, 57, 5425. (g) Musser, C. A.; Richey, H. G. J. Org. Chem. 2000, 65, 7750. (h) Reddy, L. R.; Hu, B.; Prashad, M.; Prasad, K. Org. Lett. 2008, 10, 3109.

5. (a) Kwak, Y.-S.; Corey, E. J. Org. Lett. 2004, 6, 3385. (b) Shen, K.-H.; Yao, C.-F. J. Org. Chem. 2006 , 71 , 3980. (c) Shen, K.-H.; Kuo, C.-W.and; Yao C.-F. Tetrahedron Lett. 2007, 48, 6348.

(d) Shen, K.-H.; Liu, J.-T.; Wu, Y.-R.; Yao C.-F. Synth. Commun. 2007, 37, 3677.

6. (a) Corey, E. J.; Naef, R.; Hannon, F. J. J. Am. Chem. Soc. 1986, 108, 7114. (b) Krause, N. Modern Organocopper Chemistry; Wiley-VCH: Weinheim, 2002. (c) Feringa, B. L. Acc. Chem. Res. 2000, 33, 346. (d) Pichota, A.; Pregosin, P. S.; Valentini, M.; Worle, M.; Seebach, D. Angew. Chem., Int. Ed. 2000, 39, 153. (e) Alexakis, A.; Benhaim, C.; Rosse, S.; Humam, M. J. Am. Chem. Soc. 2002, 124, 5262. (f) Xie, X. L.; Auel, C.; Henze, W.; Gschwind, R. M. J. Am. Chem. Soc. 2003, 125, 1595. (g) Hird, A. W.; Hoveyda, A. H. Angew. Chem. Int. Ed. 2003, 42, 1276. (h) Yoshihiro Miyake, Mo Wu, M. Jalilur Rahman, Yoshiyuki Kuwatani, and Masahiko Iyoda J. Org. Chem. 2006, 71, 6110. (i) Ghosh, P.; Lotesta, S. D.; Williams, L. J. J. Am. Chem. Soc. 2007, 129, 2438. (j) Hainke, S.; Singh, I.; Hemmings, J.; Seitz, O. J. Org. Chem. 2007, 72, 8811. (k) Usui, S.; Hashimoto, Y.; Morey, J. V.; Wheatley, A. E. H.; Uchiyama, M. J. Am. Chem. Soc. 2007, 129, 15102. (1) Boudet, N.; Dubbaka, S. R.; Knochel, P. Org. Lett. 2008, 10,1715 .

7. (a) Chong, J. M.; Shen, L.; Taylor, N. J. J. Am. Chem. Soc. 2000, 122, 1822. (b) Pellegrinet, S. C.; Goodman, J. M. J. Am. Chem. Soc. 2006, 128, 3116. (c) Christinat, N.; Scopelliti, R.; Severin, K. J. Org. Chem. 2007, 72, 2192. (d) Hercouet, A.; Berree, F.; Lin, C. H.; Toupet, L.; Carboni, B. Org. Lett. 2008, 9, 1717. (e) Dias, L. C.; Marchi, A. A. d.; Ferreira, M. A. B.; Aguilar, A. M. Org. Lett. 2007, 9, 4869. (f) Paton, R. S.; Goodman, J. M. J. Org. Chem. 2008, 73, 1253. (g) Dias, L. C.; Marchi, A. A. D.; Ferreira, M. A. B.; Aguilar, A. M. J. Org. Chem. 
2008, 73, 6299. (h) Beenen, M. A.; An, C.; Ellman, J. A. J. Am. Chem. Soc. 2008, 130, 6910. (i) Sumida, Y.; Yorimitsu, H.; Oshima, K. Org. Lett. 2008, 10, 4677.

8. (a) Jeon, Y. T.; Lee, C.-P.; Mariano, P. S. J. Am. Chem. Soc. 1991, 113, 8847. (b) Sibi, M. P.; Ji, J. J. Am. Chem. Soc. 1996, 118, 9200. (c) Sibi, M. P.; Ji, J.; Sausker, J. B.; Jasperse, C. P. J. Am. Chem. Soc. 1999, 121, 7517. (d) Mirakata, M.; Tsutsui, H.; Hoshino, O. Org. Lett. 2001, 3, 299. (e) Sibi, M. P.; Manyem, S. Org. Lett. 2002, 4, 2929. (f) Sibi, M. P.; Liu, P.; Ji, J.; Hajra, S.; Chen, J.-X. J. Org. Chem. 2002, 67, 1738. (g) Srikanth, G. S. C.; Castle, S. L. Org. Lett. 2004, 6, 449. (h) He, L.; Srikanth, G. S. C.; Castle. S. L. J. Org. Chem. 2005, 70, 8140. (i) Lee, S.; Lim, C. J.; Kim, S.; Subramaniam, R.; Zimmerman, J.; Sibi, M. P. Org. Lett. 2006, 8, 4311. (j) Fukuyama, T.; Kobayashi, M.; Kamata, Md. T. R. N.; Ryu, I. Org. Lett. 2006, 8, 4311.

9. For books and reviews, see: (a) Giese, B. In Radicals in Organic Synthesis: Formation of Carbon-Carbon Bonds; Pergamon: Oxford, 1986. (b) Motherwell, W. B.; Crich, D. In Free Radical Chain Reactions in Organic Synthesis; Academic Press: London, 1992. (c) Fossey, J.; Lefort, D.; Sorba, J. In Free Radicals in Organic Synthesis; Wiley: Chichester, 1995. (d) Renaud, P.; Sibi, M. P. In Radicals in Organic Synthesis; Wiley-VCH: Weinheim, 2001; Vols. 1 and 2.

(e) Ollivier, C.; Renaud, P. Chem. Rev. 2001, 101, 3415. (f) Alcaide, B.; Almendros, P.; Aragoncillo, C.; Redondo, M. C. J. Org. Chem. 2007, 72, 1604. (g) Ryu. L.; Uehara, S.; Hirao, H, Fukuyama, T. Org. Lett. 2008, 10, 1005. (h) Yamada, K.-I. Nakano, M.; Maekawa, M.; Akindele, T.; Tomioka, K. Org. Lett. 2008, 10, 3805. (i) Viswanathan, R.; Smith, C. R.; Prabhakaran, E. N.; Johnston, J. N. J. Org. Chem. 2008, 73, 3040.

10.(a) Bernasconi, C. F.; Ketner, R. J. J. Org. Chem. 1998, 63, 6266. (b) Bernasconi, C. F.; Ketner, R. J.; Ragains, M. L.; Chen, X.; Rappoport, Z. J. Am. Chem. Soc. 2001, 123, 2155. (c) Bug, T.; Mayr, H. J. Am. Chem. Soc. 2003, 125, 12980. (d) Xu, F.; Armstrong III, J. D.; Zhou, G. X.; Simmons, B.; Hughes, D.; Ge, Z.; Grabowski, E. J. J. J. Am. Chem. Soc. 2004, 126, 13002. (e) Kaupp, G.; Naimi-Jamal, M. R.; Schmeyers, J. Tetrahedron 2003, 59, 3753. (f) Cochard, F.; Laronze, M.; Sigaut, P.; Sapi, J.; Laronze, J.-Y. Tetrahedron Lett. 2004, 45, 1703. (g) Shaabani, A.; Bazgir, A. Tetrahedron Lett. 2004, 45, 2575. (h) Akue-Gedu, R.; Henichart, J.-P.; Couturierc, D.; Rigo, B. Tetrahedron Lett 2004, 45, 9197. (i) Deb, M. L.; Bhuyan, P. J. Tetrahedron Lett. 2005, 46, 6453. (j) Sabitha, G.; Kumar, M. R.; Reddy, M. S. K.; Yadav, J. S.; Krishna, K. V. S. R.; Kunwar, A. C. Tetrahedron Lett. 2005, 46, 1659. (k) Dumas, A. M.; Seed, A.; Zorzitto, A. K.; Fillion, E. Tetrahedron Lett. 2007, 48, 7072. (1) Wang, X.-S.; Zhang, M.-M.; Jiang, H.; Yaoa, C.-S.; Tua, S.-J.Tetrahedron 2007, 63, 4439.

11.(a) Sorensen, U. S.; Falch, E.; Krogsgaard-Larsen, P. J. Org. Chem. 2000, 65, 1003. (b) Hin, B.; Majer, P.; Tsukamoto, T. J. Org. Chem. 2002, 67, 7365. (c) Snyder, C. A.; Selegue, J. P.; Dosunmu, E.; Tice, N. C.; Parkin, S. J. Org. Chem. 2003, 68, 7455. (d) Halton, B.; Dixon, G. M.; Jones, C. S.; Parkin, C. T.; Veedu, R. N.; Bornemann, H.; Wentrup, C. Org. Lett. 2005, 7, 949. (e) George, L.; Veedu, R. N.; Sheibani, H.; Taherpour, A. A.; Flammang, R.; Wentrup, C. J .Org. Chem. 2007, 72, 1399. (f) Sharma, A. K.; Subramani, A. V.; Gorman, C. B. Tetrahedron 2007, 63, 389.

12.(a) Emtenas, H.; Alderin, L.; Almqvist, F. J. Org. Chem. 2001, 66, 6756. (b) Raillard, S. P.; Chen, W.; Sullivan, E.; Bajjalieh, W.; Bhandari, A.; Baer, T. A. J. Comb. Chem. 2002, 4, 470. 
(c) Giacomelli, G.; Porcheddu, A.; Salaris, M.; Taddei, M. Eur. J. Org. Chem. 2003, 537. (d) Pemberton, N.; Jakobsson, L.; Almqvist, F. Org. Lett. 2006, 8, 935.

13. (a) Watanabe, T.; Knopfel, T. F.; Carreira, E.M. Org. Lett. 2003, 5, 4557. (b) Fillion, E.; Wilsily, A. J. Am. Chem. Soc. 2006, 128, 2774. (c) Knopfel, T. F.; Zarotti, P.; Ichikawa, T.; Carreira, E. M. J. Am. Chem. Soc. 2005, 127, 9682. (d) Knopfel, T. F.; Carreira, E. M. J. Am. Chem. Soc. 2003, 125, 6054. (e) Fillion, E.; Dumas, A. M.; Kuropatwa, B. A. Malhotra, N. R.; Sitler, T. C. J. Org. Chem. 2006, 71, 409. (f) Fillion, E.; Dumas, A. M.; Hogg, S. A. J. Org. Chem. 2006, 71, 9899. (g) Perreault, S.; Spino, C. Org. Lett. 2006, 8, 4385. (h) George, L.; Veedu, R. N.; Sheibani, H.; Taherpour, A. A.; Flammang, R.; Wentrup, C. J. Org. Chem. 2007, 72, 1399. (i) Kaumanns, O.; Mayr, H. J. Org. Chem. 2008, 73, 2738.

14.(a) Fillion, E.; Fishlock, D. Org. Lett. 2003, 5, 4653. (b) Fillion, E.; Fishlock, D.; Wilsily, A.; Goll, G. M. J. Org. Chem. 2005, 70, 1316.

15.(a) Yao, C.-F.; Chu, C.-M.; Liu, J.-T. J. Org. Chem. 1998, 63, 719. (b) Chu, C.-M.; Liu, J.-T.; Lin, W.-W.; Yao, C.-F. J. Chem. Soc., Perkin Trans. 1, 1999, 47. (c) Liu, J.-Y.; Liu, J.-T., Yao, C.-F. Tetrahedron Lett. 2001, 42, 3613. (d) Liu, J.-Y.; Jang, Y.-J.; Lin, Shih, Y.-K.; Hu, S.-R.; Chu, C.-M.; Yao, C.-F. J. Org. Chem. 2001, 66, 6021. (e) Liu, J.-Y.; Jang, Y.-J.; Lin, W.-W.; Liu, J.-T.; Yao, C.-F. J. Org. Chem. 2003, 68, 4030. (f) Liu, J.-T.; Yao, C.-F. Tetrahedron Lett. 2001, 42, 6147. (g) Tu, Z.; Lin, C.; Jang, Y.; Jang, Y.-J.; Ko, S.; Fang, H.; Liu, J.-T.; Yao, C.-F. Tetrahedron Lett. 2006, 47, 6133. 